\title{
The risk of coronary heart disease after diagnosis of gallbladder polyp: a retrospective nationwide population-based cohort study
}

\author{
Chien-Hua Chen ${ }^{1,2,3}$, Cheng-Li Lin ${ }^{4,5}$, Chia-Hung Kao ${ }^{6,7,8}$ \\ ${ }^{1}$ Digestive Disease Center, Changbing Show-Chwan Memorial Hospital, Lukang Township, Changhua; ${ }^{2}$ Digestive Disease Center, Show-Chwan \\ Memorial Hospital, Changhua; ${ }^{3}$ Department of Food Science and Technology, Hungkuang University, Taichung; ${ }^{4}$ Management Office for Health \\ Data, China Medical University Hospital, Taichung; ${ }^{5}$ College of Medicine, ${ }^{6}$ Graduate Institute of Biomedical Sciences and School of Medicine, \\ College of Medicine, China Medical University, Taichung; ${ }^{7}$ Department of Nuclear Medicine and PET Center, and Center of Augmented \\ Intelligence in Healthcare, China Medical University Hospital, Taichung; ${ }^{8}$ Department of Bioinformatics and Medical Engineering, Asia University, \\ Taichung \\ Contributions: (I) Conception and design: CH Chen, CH Kao; (II) Administrative support: CH Kao; (III) Provision of study materials or patients: \\ None; (IV) Collection and assembly of data: All authors; (V) Data analysis and interpretation: All authors; (VI) Manuscript writing: All authors; \\ (VII) Final approval of manuscript: All authors. \\ Correspondence to: Chia-Hung Kao, MD. Professor, Graduate Institute of Biomedical Sciences and School of Medicine, China Medical University, \\ No. 2, Yuh-Der Road, Taichung 404. Email: d10040@mail.cmuh.org.tw; dr.kaochiahung@gmail.com.
}

Background: To assess the subsequent risk of coronary heart disease (CHD) after the diagnosis of gallbladder polyp (GP).

Methods: We identified 2,815 GP patients aged $\geq 20$ years from the Longitudinal Health Insurance Database between 2000 and 2011 and followed up the patients until the occurrence of CHD or the end of 2011, the patient would be censored in the occurrence of death, missed information, or withdrawal from the NHI. We selected 11,260 non-GP subjects by 4:1 randomly matching with the case cohort according to age, sex, and index date of GP diagnosis.

Results: GP cohort had greater risk of CHD than the control cohort [11.1 vs. 8.07 per 1,000 person-y, adjusted HR (aHR) of 1.28, 95\% confidence interval (CI), 1.07-1.53] after adjusting age, sex, hypertension, diabetes, hyperlipidemia, gallstone, chronic obstructive pulmonary disease, and arrhythmia. The risk of CHD was significantly higher in the non-cholecystectomy cohort of GP patients than that in the non-GP cohort (10.9 vs. 8.07 per 1,000 person-y; aHR =1.28; 95\% CI, 1.06-1.55). However, the risk of CHD contributed by GP was not significant after cholecystectomy (12.3 vs. 8.07 per 1,000 person-y; aHR =1.24; $95 \%$ CI, $0.83-$ 1.85). Compared with the non-GP cohort without hypertension, the risk of CHD increased for GP cohort without ( $\mathrm{aHR}=1.48 ; 95 \% \mathrm{CI}, 1.18-1.87$ ) or with hypertension (aHR =3.00; 95\% CI, 2.30-3.92). Compared with the non-GP cohort without diabetes, the risk of CHD increased for GP cohort without diabetes $(\mathrm{aHR}=$ 1.46; 95\% CI, 1.21-1.76) or with diabetes (aHR =2.07; 95\% CI, 1.35-3.18). Compared with the non-GP cohort without hyperlipidemia, the risk of CHD increased for GP cohort without (aHR =1.37; 95\% CI, 1.10-1.70) or with hyperlipidemia ( $\mathrm{aHR}=2.63$; 95\% CI, 2.01-3.44). Compared with the non-GP cohort without arrhythmia, the risk of CHD for GP patients increased without (aHR =1.40; 95\% CI, 1.17-1.69) or with arrhythmia (aHR =2.88; 95\% CI, 1.82-4.57).

Conclusions: GP is associated with increased risk of developing CHD, and the risk increases with the presence of coexisting hypertension, diabetes, hyperlipidemia, or arrhythmia.

Keywords: Coronary heart disease (CHD); gallbladder polyp (GP); cholecystectomy

Submitted Sep 07, 2019. Accepted for publication Nov 08, 2019.

doi: 10.21037/atm.2019.11.114

View this article at: http://dx.doi.org/10.21037/atm.2019.11.114 


\section{Introduction}

Gallbladder polyp (GP) is diagnosed based on the presence of soft tissue mass within the gallbladder wall, and ultrasonography has over $90 \%$ of sensitivity and specificity in ascertaining the diagnosis of GP (1-3). GPs are classified into true polyps and pseudopolyps; the former include adenomas and adenocarcinomas, and the latter mainly include cholesterol polyps and inflammatory polyps. About $70 \%$ of GPs are cholesterol polyps, and the prevalence of true GPs is only approximately $4-7 \%$. Most GPs are clinically diagnosed based on ultrasonography and only few undergo surgery to have histopathological confirmation. Most studies of GPs have focused on the prevalence or the associated risk factors; nevertheless, the clinicians are mainly concerned about the indication for surgery although the malignant potential of GP is quite low (4-6).

The prevalence of GPs for Chinese is approximately $6.9-9.5 \%$, which is greater than that for Danish and Japanese populations (5-9). The pathophysiological mechanisms for gallbladder stones and GPs are different. The reported risk factors for gallbladder stones include aging, sex (female), race, a family history of gallbladder stones, obesity, diabetes, and hypertriglyceridemia (10-12). In the contrast, GPs are generally suggested to be related to sex (male), glucose intolerance, obesity, or metabolic syndrome. Moreover, it remains debated about the role of chronic hepatitis B virus (HBV) infection as the risk factor for GPs (5-9,13-17). The variation of race, diet, and environmental characteristics may contribute to the inconsistent conclusions about the risk factors for GPs. The association between metabolic disorders and GPs might portend the association of GP with cardiovascular disease $(5,13,16,17)$. However, most GP literature focuses on cross-sectional associations and cohort studies are therefore needed, although our former cohort study has evidenced the close association of GP with stroke (18).

Coronary heart disease (CHD) constitutes a considerable global public health burden, thus it is critical to identify its associated risk factors for the prevention of this potentially fatal disease. The contribution of metabolic syndrome to the development CHD has been consistently documented in Western and Eastern areas (19-23). Furthermore, the reported prevalence of CHD has also increased in AsiaPacific areas, including Taiwan, due to the increasing prevalence of sedentary lifestyles, unhealthy dietary habits, and metabolic disorders (19).

With the wide application of ultrasonography, the reported prevalence of GP may increase. Since GPs and CHD share several common risk factors, it is warranted to assess the association between CHD and GP for the prevention of CHD. We conducted a nationwide population-based cohort study by analyzing data from a nationwide medical database, namely the National Health Insurance Research Database (NHIRD), to assess the association between GP and subsequent risk of CHD development. We also assessed the contribution of cholecystectomy to the risk of CHD for the GP patients.

\section{Methods}

\section{Data source}

The Taiwan National Health Insurance (NHI) program has started since 1995 and this mandatory governmentmonopolized program has covered more than $99.2 \%$ of the residents in Taiwan (24). The National Health Research Institutes (NHRI), which owns many good statisticians and computer experts, is the largest health research institute in Taiwan and has been authorized by the government to establish NHIRD for storing the database of NHI program. The NHRI is responsible for the provision of appropriate data files to the researchers. We obtained the Longitudinal Health Insurance Database 2000 (LHID 2000), a database containing the claims data for hospitalization for one million people from 1996 to 2011, randomly sampled from 2000 NHIRD enrollment records after application to the NHRI. The distributions of sex, age and health care cost between the one million population of the LHID 2000 and the 23 million population of the NHI program were similar. All the data have been de-identified and the diagnostic codes in the LHID 2000 were based on the International Classification of Disease, Ninth Revision, Clinical Modification (ICD-9-CM). The NHRI assigned a random number for each person by Knuth and Park and Miller's method $(25,26)$.

\section{Data availability statement}

Any researcher can access this data after approval from the Taiwan Ministry of Health and Welfare (MOHW), and the patient consent is exempted due to de-identification of the NHIRD (27). The contact information for MOHW is: Taiwan Ministry of Health and Welfare Address: No. 488, Sec. 6, Zhongxiao E. Rd., Nangang Dist., Taipei City 115. Phone: +886-2-8590-6848. Email: stcarolwu@mohw.gov.tw. 


\section{Ethics statement}

The authors are accountable for all aspects of the work in ensuring that questions related to the accuracy or integrity of any part of the work are appropriately investigated and resolved. The Institutional Review Board (IRB) of China Medical University (CMUH104-REC2-115-CR4) has approved this study and waived the consent requirement.

\section{Sampled participants}

Retrieved from the LHID 2000, the GP cohort consisted of patients aged $\geq 20$ years who had been diagnosed with GPs (ICD-9-CM code 575.6) between 2000 and 2011. For each GP case, 4 patients without GP matched by age (every 5 -y span), sex, and index date year were randomly selected from the LHID 2000 as the non-GP cohort. With the date of GP diagnosis defined as the index date, this study excluded the individuals diagnosed with CHD (ICD-9-CM codes 410414) before the index date, with missing information, or aged $<20$ years. The comorbidities considered for analysis in this study included hypertension (ICD-9-CM codes 401-405), diabetes (ICD-9-CM code 250), hyperlipidemia (ICD-9-CM code 272), congestive heart failure (CHF; ICD-9-CM code 428), alcohol-related illness (ICD-9-CM codes 291, 303, 305, 571.0-571.3, 790.3, A215, and V11.3), gallstone (ICD-9-CM code 574), obesity (ICD-9-CM code 278), chronic obstructive pulmonary disease (COPD) (ICD9-CM codes 491, 492, and 496), and arrhythmia (ICD-9CM codes 426 and 427).

\section{Outcomes}

CHD was the primary endpoint, and each patient was followed until the occurrence of CHD or the end of 2011, the patient would be censored due to death, missed information, withdrawal from the NHI. The diagnoses of CHD included acute myocardial infarction (ICD-9-CM codes 410), acute or subacute forms of ischemic heart disease (ICD-9-CM codes 411), old myocardial infarction (ICD-9CM code 412), angina pectoris (ICD-9-CM code 413), and chronic ischemic heart disease (ICD-9-CM code 414).

\section{Statistical analysis}

We compared the distributions of categorical variables by the chi-square test and examined the differences of continuous variables by the Student $t$-test. A Kaplan-Meier curve was plotted to show the cumulative incidence of $\mathrm{CHD}$, and the differences between the cohorts was analyzed by the log-rank test. The incidence densities of CHD were expressed as person-years for the cohorts. Univariate and multivariate Cox proportional hazards models were used for the expression of hazard ratios (HRs) and $95 \%$ confidence intervals (CIs). We used a test of scaled Schoenfeld residuals to examine the proportional hazard model assumption. The proportionality assumption would be violated if the results of the test revealed a significant relationship between Schoenfeld residuals for GP and follow-up time $(\mathrm{P}$ value $=$ $0.01)$. In the subsequent analyses, we stratified the follow-up duration to deal with the violation of proportional hazard assumption. The risk factors for adjustment in multivariate Cox models included age sex, and comorbidities of hypertension, diabetes, hyperlipidemia, gallstone, COPD, and arrhythmia. The software used for statistical analyses was SAS version 9.4 (SAS Institute Inc., Cary, NC, USA), and a 2 -sided test with a $\mathrm{P}$ value $<0.05$ was considered statistically significant.

\section{Results}

Table 1 presents the baseline demography of the cohorts with and without GP. The GP cohort consisted of 2,815 patients (mean age $46.2 \pm 13.4$ ) and the non-GP

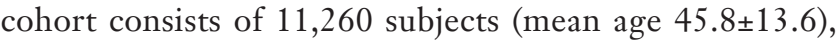
respectively. In both cohorts, $59.1 \%$ of the patients were male and most patients were aged $\leq 49$ years $(63.4 \%)$. The GP cohort had a higher prevalence of hypertension, diabetes, hyperlipidemia, alcohol-related illness, gallstone, obesity, COPD, and arrhythmia than the non-GP cohort (all $\mathrm{P}<0.05$ ). The mean follow-up period for the GP cohort and non-GP cohort was $5.42 \pm 3.28$ and $5.65 \pm 3.24$ years, respectively. Figure 1 illustrates that GP cohort had higher cumulative incidence of CHD than the non-GP cohort (log-rank test, $\mathrm{P}<0.001)$.

Table 2 compares the incidence and HR of CHD between the GP and non-GP cohorts stratified by sex, age, and the presence of comorbidity. The GP cohort had a higher risk of CHD [adjusted HR $(\mathrm{aHR})=1.28$; 95\% CI, 1.07-1.53], with an incidence of 11.1/1,000 person-y incidence for the GP cohort and 8.07/1,000 person-y for non-GP cohort after adjustment for age, sex, and comorbidities of hypertension, diabetes, hyperlipidemia, gallstone, COPD, and arrhythmia. The contribution of GP to the development of CHD was greater in men $(\mathrm{aHR}=1.33 ; 95 \% \mathrm{CI}, 1.06-1.66)$, but not significant in women $(\mathrm{aHR}=1.18 ; 95 \% \mathrm{CI}, 0.87-1.60)$. 
Table 1 The baseline demography of the cohorts with and without GP

\begin{tabular}{|c|c|c|c|}
\hline \multirow{3}{*}{ Variable } & \multicolumn{2}{|c|}{ Gallbladder polyp } & \multirow{3}{*}{$P$ value } \\
\hline & No & Yes & \\
\hline & $\mathrm{N}=11,260(\%)$ & $\mathrm{N}=2,815(\%)$ & \\
\hline Gender & & & 0.99 \\
\hline Female & 4,608 (40.9) & $1,152(40.9)$ & \\
\hline Male & $6,652(59.1)$ & $1,663(59.1)$ & \\
\hline Stratify age & & & 0.99 \\
\hline$\leq 34$ & $2,424(21.5)$ & $606(21.5)$ & \\
\hline $35-49$ & $4,712(41.9)$ & $1,178(41.9)$ & \\
\hline $50-64$ & $3,068(27.3)$ & 767 (27.3) & \\
\hline $65+$ & $1,056(9.38)$ & $264(9.38)$ & \\
\hline Age, mean $(\mathrm{SD})^{\#}$ & $45.8(13.6)$ & $46.2(13.4)$ & 0.20 \\
\hline \multicolumn{4}{|l|}{ Comorbidity } \\
\hline Hypertension & $1,881(16.7)$ & $551(19.6)$ & $<0.001$ \\
\hline Diabetes & 607 (5.39) & $193(6.86)$ & 0.003 \\
\hline Hyperlipidemia & $1,351(12.0)$ & $576(20.5)$ & $<0.001$ \\
\hline Alcohol-related illness & $372(3.30)$ & $189(6.71)$ & $<0.001$ \\
\hline Gallstone & 190 (1.69) & $383(13.6)$ & $<0.001$ \\
\hline Obesity & $129(1.15)$ & $50(1.78)$ & 0.008 \\
\hline COPD & $581(5.16)$ & $248(8.81)$ & $<0.001$ \\
\hline Arrhythmia & $355(3.15)$ & $134(4.76)$ & $<0.001$ \\
\hline
\end{tabular}

Chi-square test; ", 2-sample $t$-test. GP, gallbladder polyp; COPD, chronic obstructive pulmonary disease.

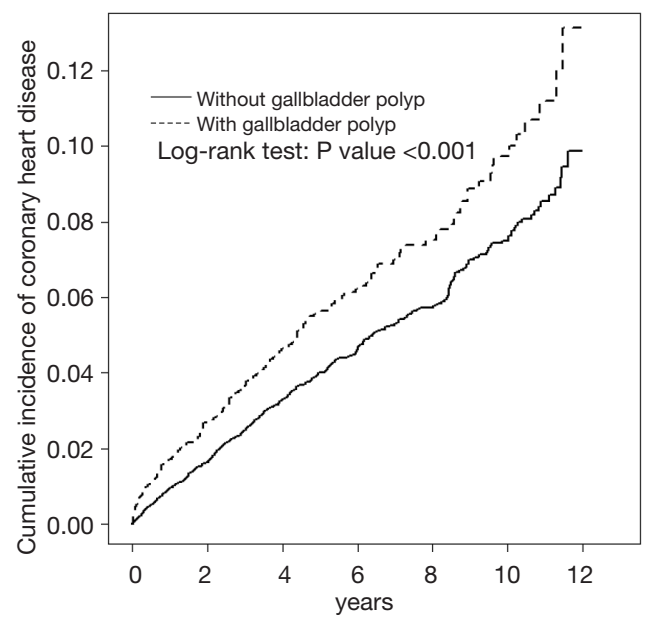

Figure 1 Cumulative incidence of coronary heart disease for patients with and without gallbladder polyp.
The patients aged less than 34 years with GP had a 3.02-fold higher risk of CHD than the non-GP cohort (aHR $=3.02$; 95\% CI, 1.28-7.11). The patients aged 35-49 years with GP had a 1.51-fold higher risk of CHD than nonGP cohort (aHR $=1.51 ; 95 \%$ CI, 1.10-2.08). The patients aged more than 65 years with GP had a 1.48 -fold higher risk of $\mathrm{CHD}$ than the non-GP cohort $(\mathrm{aHR}=1.48 ; 95 \%$ CI, 1.01-2.16). It was noted that the contribution of GP to the development of CHD was greater for patients without comorbidity ( $\mathrm{aHR}=1.43 ; 95 \% \mathrm{CI}, 1.03-1.97$ ) than those with comorbidity ( $\mathrm{aHR}=1.07$; 95\% CI, 0.87-1.33).

Within $\leq 3$ years of follow-up, the GP cohort had a higher risk of CHD than the non-CP cohort $(\mathrm{aHR}=1.36$; 95\% CI, 1.21-1.52). Moreover, the risk of CHD in the CP cohort was consistently higher than that in the non-CP cohort after more than 3 years of follow-up ( $\mathrm{aHR}=1.22$; 95\% CI, 1.08-1.37).

Table 3 presents the HRs of CHD based on age, sex, and comorbidities in the univariate and multivariate Cox regression models. Compared with patients aged $\leq 34$ years, the risk of CHD was 1.27-fold higher in those aged $35-49$ years (95\% CI, 1.06-1.52), 3.57-fold higher in those aged 50-64 years (95\% CI, 2.36-5.42), and 6.79-fold higher in those aged $\geq 65$ years (95\% CI, 4.48-10.3). The aHR of CHD was 1.33 -fold greater for men than women (95\% CI, 1.14-1.55). Besides, the multivariable models also showed that CHD was independently associated with hypertension (aHR $=1.99 ; 95 \% \mathrm{CI}, 1.67-2.37)$, diabetes $(\mathrm{aHR}=1.28 ; 95 \% \mathrm{CI}$, 1.02-1.61), hyperlipidemia (aHR $=1.55 ; 95 \%$ CI, $1.29-1.86$ ), COPD (aHR $=1.34 ; 95 \%$ CI, 1.06-1.70), and arrhythmia (aHR $=1.99 ; 95 \%$ CI, 1.54-2.57).

Table 4 shows the risk of CHD by the Cox proportional hazards regression analysis based on the status of GP and coexisting comorbidities. Compared with the non-GP patients without hypertension, the risk of CHD for GP patients without hypertension increased to 1.48 (95\% CI, 1.18-1.87) and the risk increased to 3.00 (95\% CI, 2.30 $3.92)$ in the presence of coexisting hypertension. Compared with the non-GP patients without diabetes, the risk of CHD for GP patients without diabetes increased to 1.46 (95\% CI, 1.21-1.76) and the risk increased to 2.07 (95\% CI, 1.35-3.18) in the presence of coexisting diabetes. Compared with the non-GP patients without hyperlipidemia, the risk of CHD for GP patients without hyperlipidemia increased to 1.37 (95\% CI, 1.10-1.70) and the risk increased to 2.63 (95\% CI, 2.01-3.44) in the presence of coexisting hyperlipidemia. Compared with the non-GP patients 
Table 2 The comparison of the incidence and HR of CHD between the GP and non-GP cohorts stratified by sex, age, and the presence of comorbidity

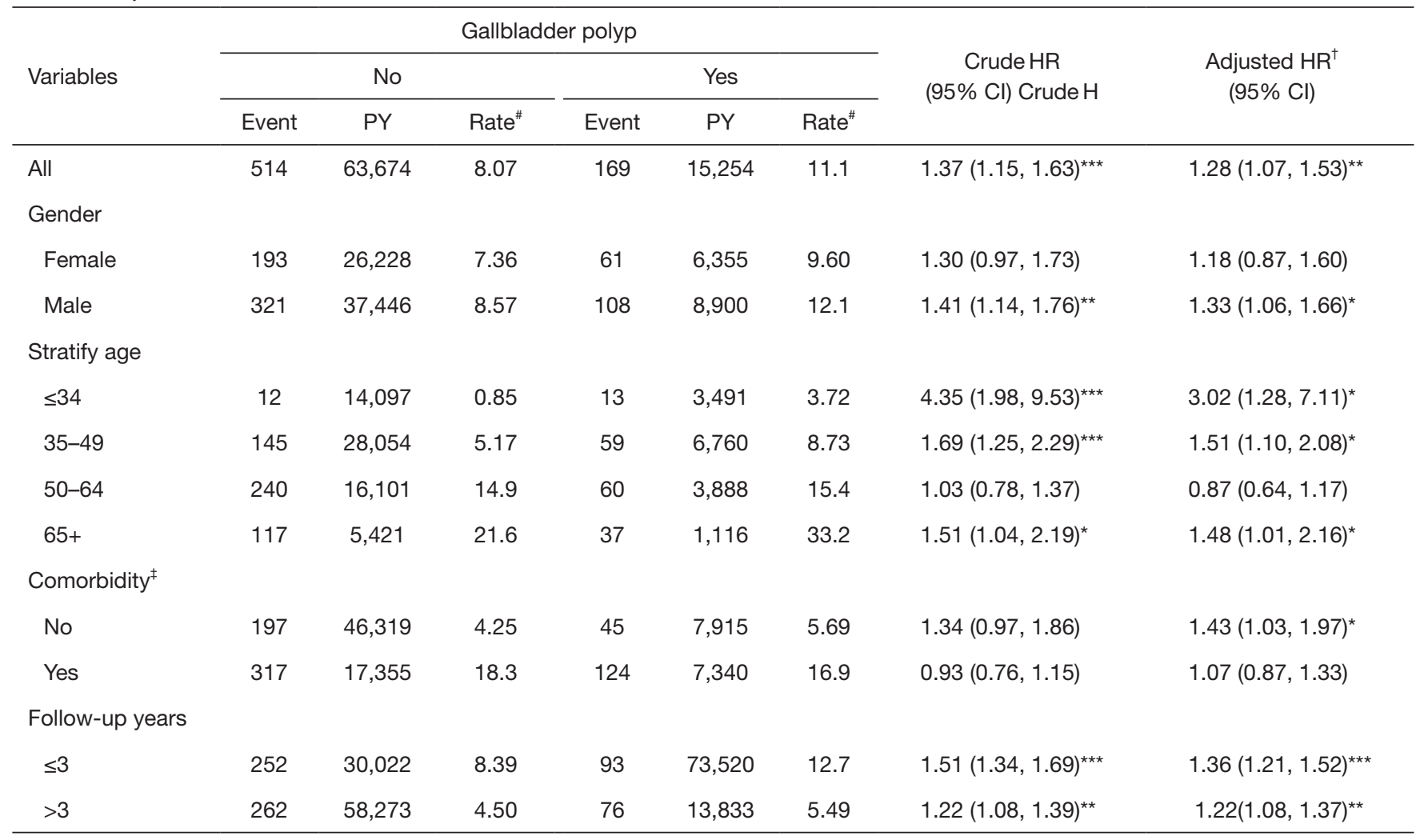

\#, incidence rate per 1000 person-years; ${ }^{\dagger}$, multivariate analysis including age, sex, and comorbidities of hypertension, diabetes, hyperlipidemia, gallstone, COPD, and arrhythmia; Comorbidity ${ }^{\ddagger}$, patients with a comorbidity of hypertension, diabetes, hyperlipidemia, alcohol-related illness, gallstone, obesity, COPD, or arrhythmia were classified in the comorbidity group; *, $\mathrm{P}<0.05 ;{ }^{* *}, \mathrm{P}<0.01$; ${ }^{* \star}$, $\mathrm{P}<0.001$. PY, person-years; Crude HR, relative hazard ratio; CHD, coronary heart disease; GP, gallbladder polyp; COPD, chronic obstructive pulmonary disease.

without arrhythmia, the risk of CHD for GP patients without arrhythmia increased to 1.40 (95\% CI, 1.17-1.69) and the risk increased to 2.88 (95\% CI, 1.82-4.57) in the presence of coexisting arrhythmia.

Table 5 shows the risk of CHD in patients with GP based on the status of cholecystectomy and in patients without GP. The risk of CHD was significantly higher in the noncholecystectomy cohort of GP patients than that in the non-GP cohort (10.9 vs. 8.07 per 1000 person-y), with an aHR of 1.28 (95\% CI, 1.06-1.55) (Table 5). However, the risk of CHD contributed by GP was not significant after cholecystectomy (12.3 vs. 8.07 per 1,000 person-y; aHR = 1.24; $95 \%$ CI, 0.83-1.85).

\section{Discussion}

To our knowledge, our population-based study is the first to demonstrate the risk of CHD will increase after diagnosis of GP. Most former GP-associated studies focused on the prevalence or risk factors of GP alone, even though some risk factors for CHD have been found to be related to the development of GP (5-9,13-15). The use of a nationwide database with a large population and the 12-year observation enhanced the statistical power and provided the generalization in Taiwan.

In contrast to gallstones, our study showed that GP had the predilection of male gender. Estrogen may protect from the development of GP since it can impair the esterification of cholesterol by reducing the activity of both hepatic and intestinal acyl-CoA: cholesterol acyltransferase 2 (ACAT2), an enzyme facilitating the pinocytosis of cholesterol and papillary hyperplasia in the gallbladder mucosa (28).

Hyperlipidemia, hypertension, gallstones, and diabetes were the most common comorbidities for GP, and our 
Table 3 Incidence and hazard ratio for CHD and CHD-associated risk factor

\begin{tabular}{|c|c|c|c|c|c|}
\hline Variable & Event & PY & Rate $^{\#}$ & Crude HR $(95 \% \mathrm{Cl})$ & Adjusted $\mathrm{HR}^{\dagger}(95 \% \mathrm{Cl})$ \\
\hline No & 514 & 63,674 & 8.07 & 1.00 & 1.00 \\
\hline Yes & 169 & 15,254 & 11.1 & $1.37(1.15,1.63)^{\star \star \star}$ & $1.28(1.07,1.53)^{\star \star}$ \\
\hline \multicolumn{6}{|l|}{ Age, year } \\
\hline $35-49$ & 204 & 34,814 & 5.86 & $4.10(2.71,6.22)^{\star \star \star}$ & $1.27(1.06,1.52)^{\star \star *}$ \\
\hline $50-64$ & 300 & 19,989 & 15.0 & $10.5(6.98,15.8)^{\star \star \star}$ & $3.57(2.36,5.42)^{\star \star \star}$ \\
\hline $65+$ & 154 & 6,537 & 23.6 & $16.4(10.8,25.1)^{\star \star \star}$ & $6.79(4.48,10.3)^{\star \star \star}$ \\
\hline \multicolumn{6}{|l|}{ Sex } \\
\hline \multicolumn{6}{|c|}{ Hypertension } \\
\hline No & 385 & 66,644 & 5.78 & 1.00 & 1.00 \\
\hline Yes & 298 & 12,284 & 24.3 & $4.19(2.60,4.87)^{\star \star \star}$ & $1.99(1.67,2.37)^{\star \star \star}$ \\
\hline \multicolumn{6}{|c|}{ Diabetes } \\
\hline No & 584 & 75,014 & 7.79 & 1.00 & 1.00 \\
\hline Yes & 99 & 3,914 & 25.3 & $3.24(2.61,4.01)^{\star \star \star}$ & $1.28(1.02,1.61)^{\star}$ \\
\hline \multicolumn{6}{|c|}{ Hyperlipidemia } \\
\hline No & 472 & 69,209 & 6.82 & 1.00 & 1.00 \\
\hline No & 644 & 75,868 & 8.49 & 1.00 & 1.00 \\
\hline Yes & 39 & 3,061 & 12.7 & $1.50(1.09,2.07)^{\star}$ & $0.96(0.69,1.35)$ \\
\hline \multicolumn{6}{|l|}{ Obesity } \\
\hline No & 672 & 78,114 & 8.60 & 1.00 & 1.00 \\
\hline Yes & 11 & 814 & 13.5 & $1.55(0.86,2.82)$ & \\
\hline \multicolumn{6}{|l|}{ COPD } \\
\hline No & 598 & 74,961 & 7.98 & 1.00 & 1.00 \\
\hline Yes & 85 & 3,967 & 21.4 & $2.67(2.13,3.36)^{\star \star \star}$ & $1.34(1.06,1.70)^{*}$ \\
\hline \multicolumn{6}{|c|}{ Arrhythmia } \\
\hline No & 616 & 76,546 & 8.05 & 1.00 & 1.00 \\
\hline Yes & 67 & 2,382 & 28.1 & $3.48(2.71,4.49)^{\star \star \star}$ & $1.99(1.54,2.57)^{\star \star \star}$ \\
\hline
\end{tabular}

\#, incidence rate, per 1,000 person-years; ${ }^{\dagger}$, multivariate analysis including age, sex, and comorbidities of hypertension, diabetes, hyperlipidemia, gallstone, COPD, and arrhythmia; ${ }^{*}, \mathrm{P}<0.05$; ${ }^{*}, \mathrm{P}<0.01$; ${ }^{* \star}, \mathrm{P}<0.001$. Crude HR, relative hazard ratio; $\mathrm{CHD}$, coronary heart disease; GP, gallbladder polyp; COPD, chronic obstructive pulmonary disease. 
Table 4 The risk of CHD by the Cox proportional hazards regression analysis based on the status of GP and coexisting comorbidities

\begin{tabular}{|c|c|c|c|c|c|c|}
\hline Variables & Gallbladder polyp & $\mathrm{N}$ & Event $n$ & PY & Rate $^{\#}$ & Adjusted $\mathrm{HR}^{\dagger}(95 \% \mathrm{Cl})$ \\
\hline No & No & 9,379 & 288 & 5.32 & 5.32 & 1 (Reference) \\
\hline Yes & No & 1,881 & 226 & 23.7 & 23.7 & $2.48(2.04,3.00)^{\star \star \star}$ \\
\hline No & Yes & 2,264 & 97 & 7.75 & 7.75 & $1.48(1.18,1.87)^{\star \star \star}$ \\
\hline \multicolumn{7}{|l|}{ Diabetes } \\
\hline No & No & 10,653 & 437 & 7.20 & 7.20 & 1 (Reference) \\
\hline Yes & No & 607 & 77 & 26.0 & 26.0 & $2.05(1.60,2.63)^{\star \star \star}$ \\
\hline No & Yes & 2,622 & 147 & 10.3 & 10.3 & $1.46(1.21,1.76)^{\star \star \star}$ \\
\hline No & No & 9,909 & 366 & 56,871 & 6.44 & 1 (Reference) \\
\hline Yes & No & 1,351 & 148 & 6,802 & 21.8 & $2.21(1.82,2.68)^{\star \star \star}$ \\
\hline No & Yes & 2,239 & 106 & 12,337 & 8.59 & $1.37(1.10,1.70)^{\star *}$ \\
\hline Yes & Yes & 576 & 63 & 2,917 & 21.6 & $2.63(2.01,3.44)^{\star \star \star}$ \\
\hline \multicolumn{7}{|c|}{ Arrhythmia } \\
\hline No & No & 10,905 & 466 & 61,955 & 7.52 & 1 (Reference) \\
\hline Yes & No & 355 & 48 & 1,719 & 27.9 & $2.59(1.92,3.50)^{\star * \star}$ \\
\hline No & Yes & 2,681 & 150 & 14,591 & 10.3 & $1.40(1.17,1.69)^{\star \star \star}$ \\
\hline
\end{tabular}

${ }^{\#}$, incidence rate, per 1,000 person-years; ${ }^{\dagger}$, adjusted for age and sex; ${ }^{\star \star}, \mathrm{P}<0.01 ;{ }^{\star \star \star}, \mathrm{P}<0.001$. $\mathrm{PY}$, person-years; CHD, coronary heart disease; GP, gallbladder polyp.

Table 5 The risk of CHD in patients with GP based on the status of cholecystectomy and in patients without GP

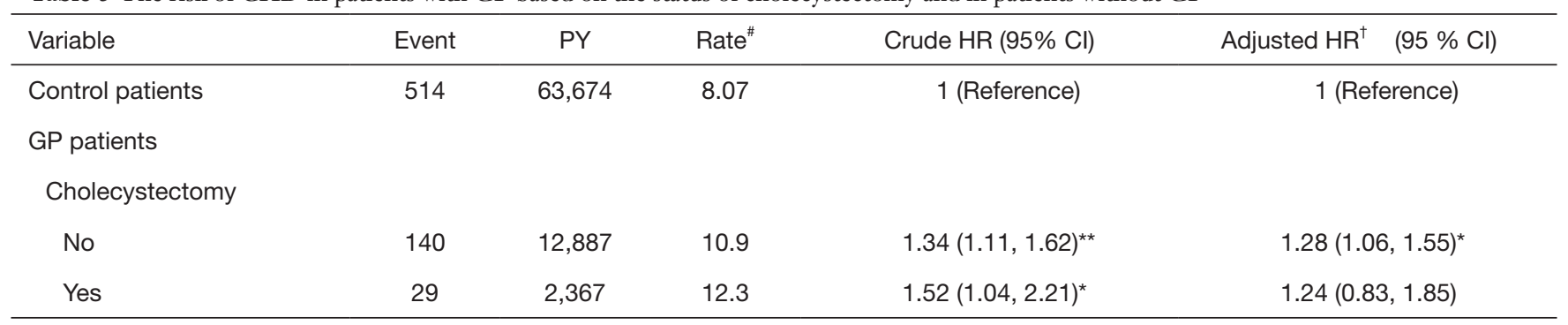

\#, incidence rate, per 1,000 person-years; ${ }^{\dagger}$, multivariate analysis including age, sex, and comorbidities of hypertension, diabetes, hyperlipidemia, gallstone, COPD, and arrhythmia; *, $\mathrm{P}<0.05$; ${ }^{* *}, \mathrm{P}<0.01$; ${ }^{* \star}, \mathrm{P}<0.001$. Crude HR, relative hazard ratio; $\mathrm{CHD}$, coronary heart disease; GP, gallbladder polyp; COPD, chronic obstructive pulmonary disease. 
findings show that GP and CHD share several common risk factors (5-9,13-15). The association between GP and CHD may be an epiphenomenon, rather than a causal effect, due to shared common risk factors. However, we have tried to minimizing the confounding effect of CHD risk factors in the multivariate Cox proportional hazards model (Table 3). In addition, the significant contribution of GP for the CHD development in the younger individuals may be due to the absence or low prevalence of CHD-associated comorbidities in the younger patients. The contribution of GP to the development of CHD was similarly greater for patients without comorbidity (Table 2). Furthermore, the cumulative incidence of CHD was higher in the GP cohort than in the non-GP cohort even though the mean follow-up period was shorter in the GP cohort (5.42 \pm $3.28 \mathrm{y})$ than in the non-GP cohort $(5.65 \pm 3.24 \mathrm{y})$ (Figure 1 ). Irrespective of the duration of follow-up, the risk of CHD was persistently for the GP cohort than that for the nonGP cohort. The risk of CHD was significantly higher in the non-cholecystectomy cohort of GP patients than that in the non-GP cohort, but the risk of CHD contributed by GP was not significant after cholecystectomy. All these findings support that GP may be a risk factor of CHD, but our results show that GP was inferior to the traditional risk factors, such as hypertension, diabetes, and hyperlipidemia, in contributing to the development of CHD (Table 4). However, it needs more studies to ascertain the to ascertain the causal effect of GP on the development of CHD due to lacking potential confounding factors and the inherent limitation of this observational study.

Some studies have suggested that the association between GP and CHD may be due to the following pathophysiological features. First, the genotype with change in the XbaI restriction fragment length polymorphisms of apolipoproteins $\mathrm{B}(\mathrm{apoB})$, predisposes to the development of GP and is a wellknown risk factor for the development of atherosclerosis (29). Furthermore, ACAT2 can esterify cholesterol in the gallbladder to facilitate the development of GP by cholesterol phagocytosis in the gallbladder mucosa and by reducing gallbladder contractility with cholesterol deposition in the gallbladder wall (28). On the other hand, ACAT2 can enhance the incorporation of cholesteryl esters into apoB in the blood to increase low-density lipoprotein particles. Second, the activation of the immune response enhances both the development of GP and atherosclerosis (30). Finally, the interactive linkage between obesity, diabetes, and inflammation can induce the development of both GP and atherosclerosis (31-33). Adipose tissue can produce adipokines to induce endothelial dysfunction and atherosclerosis by stimulating innate immune system. Furthermore, increasing insulin resistance, common for obesity and diabetes, can enhance the development of GP by reducing gallbladder contractility and modifying the bile cholesterol and can induce atherosclerosis by initiating oxidative stress and dyslipidemia.

\section{Strengths and limitations}

The present study had several strengths. First, the largescale national database provided a statistical power to our study. Second, the recruited patients were sampled from a stable population of Taiwan to provide the generalization in Taiwan. Third, our study adopted a longitudinal rather than cross-sectional approach to provide a temporal relationship between GP and CHD.

Nevertheless, our study had some limitations. First, the potential CHD-related confounding factors could not be fully assessed in this study. Second, we could not confirm the histopathological nature of polypoid lesions to make the differentiation between true polyps and pseudopolyps due to the unavailability of histopathology in the database. Most GP patients will not undergo cholecystectomy without signs of malignancy and all the literature on the epidemiology of GP made the diagnosis by using only ultrasonography. However, the accuracy of ultrasonography in diagnosing GP exceeds $90 \%$ and cholesterol polyps are assumed to be the most common type of GP $(2,3)$. Third, the diagnosis of GP identified in this study was made by the discretion of the doctors in charge of the symptomatic disease, rather than screening of the general population, based on the claims data for hospitalization. Therefore, there might be a cohort specific bias or mis-classification of asymptomatic GP patients into non-GP patients in our study. However, the possible skewed association has been minimized by assessing the risk of CHD in GP patients with and without cholecystectomy. The risk of difference was significant between the non-cholecystectomy cohort of GP patients and the non-GP cohort; nevertheless, the risk difference of CHD was comparable between the cholecystectomy cohort of GP patients and the non-GP cohort. Our findings supported that GP per se is associated the subsequent development of CHD, but we could not ascertain GP is an epiphenomenon or independent risk factor of CHD. Finally, the pathogenesis for the association between GP and CHD development could not be ascertained in our observational study. 


\section{Conclusions}

This retrospective observational study had its inherent limitation for the unavailability of possible confounding factors. This nationwide population-based cohort study revealed that GP is associated with increased risk of developing CHD and the risk increases with coexisting hypertension, diabetes, hyperlipidemia, or arrhythmia. However, GP may be less influential than other risk factors, such as hypertension, diabetes, and hyperlipidemia, in contributing to CHD development. Our findings of this study were consistent with our former literature, which similarly showed GP was inferior to the conventional risk factors in contributing to the development of stroke and the risk of GP associated stroke increased in subjects of younger age or without comorbidity (16). Further study is required to clarify whether GP is an epiphenomenon or independent risk factor for cardiovascular disease development. However, the risk of cardiovascular disease increased in GP patients with co-existing comorbidity.

\section{Acknowledgments}

Funding: This work was granted by the Ministry of Health and Welfare, Taiwan (MOHW107-TDU-B-212-123004), China Medical University Hospital (CMU106-ASIA-12, DMR-107-192), Academia Sinica Stroke Biosignature Project (BM10701010021), MOST Clinical Trial Consortium for Stroke (MOST 107-2321-B-039 -004-), Tseng-Lien Lin Foundation, Taichung, Taiwan, and Katsuzo and Kiyo Aoshima Memorial Funds, Japan.

\section{Footnote}

Conflicts of Interest: The authors have no conflicts of interest to declare.

Ethical Statement: The authors are accountable for all aspects of the work in ensuring that questions related to the accuracy or integrity of any part of the work are appropriately investigated and resolved. The Institutional Review Board (IRB) of China Medical University (CMUH104-REC2-115-CR4) has approved this study and waived the consent requirement.

\section{References}

1. Wiles R, Thoeni RF, Barbu ST, et al. Management and follow-up of gallbladder polyps. Eur Radiol 2017;27:3856-66.

2. Martin E, Gill R, Debru E. Diagnostic accuracy of transabdominal ultrasonography for gallbladder polyps: systematic review. Can J Surg 2018;61:200.

3. Mellnick VM, Menias CO, Sandrasegaran K, et al. Polypoid lesions of the gallbladder: Disease spectrum with pathologic correlation. Radiographics 2015;35:387-99.

4. Wennmacker SZ, van Dijk AH, Raessens JH, et al. Polyp size of $1 \mathrm{~cm}$ is insufficient to discriminate neoplastic and non-neoplastic gallbladder polyps. Surg Endosc 2019;33:1564-71.

5. Leng S, Zhao A, Li Q, et al. Metabolic status and lifestyle factors associated with gallbladder polyps: a covariance structure analysis. BMC Gastroenterol 2018;18:159.

6. Xu Q, Tao LY, Wu Q, et al. Prevalences of and risk factors for biliary stones and gallbladder polyps in a large Chinese population. HPB 2012;14:373-81.

7. Jørgensen T, Jensen KH. Polyps in the gallbladder: A prevalence study. Scand J Gastroenterol 1990;25:281-6.

8. Shinchi K, Kono S, Honjo S, et al. Epidemiology of gallbladder polyps: An ultrasonographic study of male self-defense officials in Japan. Scand J Gastroenterol 1994;29:7-10.

9. Segawa K, Arisawa T, Niwa Y, et al. Prevalence of gallbladder polyps among apparently healthy Japanese: Ultrasonographic study. Am J Gastroenterol 1992;87:630-3.

10. Housset C. Gallstone disease, towards a better understanding and clinical practice. Curr Opin Gastroenterol 2018;34:57-8.

11. Shabanzadeh DM, Sørensen LT, Jørgensen, T. A prediction rule for risk stratification of incidentally discovered gallstones: results from a large cohort study. Gastroenterology 2016;150:156-67.e1.

12. Di Ciaula A, Wang DQH, Portincasa P. An update on the pathogenesis of cholesterol gallstone disease. Curr Opin Gastroenterol 2018;34:71-80.

13. Park EJ, Lee HS, Lee SH, et al, Association between metabolic syndrome and gallbladder polyps in healthy Korean population. J Korean Med Sci 2013;28:876-80.

14. Yang HL, Kong L, Hou LL, et al. Analysis of risk factors for polypoid lesions of gallbladder among health examinees. World J Gastroenterol 2012;18:3015-9.

15. Chen YY, Huang WH, Yang HR. A Gallbladder Polyp in a Patient with Chronic Hepatitis B. Gastroenterology 2015;149:e3-4.

16. Chen CY, Lu CL, Chang FY, et al. Risk factors for 
gallbladder polyps in the Chinese population. Am J Gastroenterol 1997;92:2066-8.

17. Lim SH, Kim DH, Park MJ, et al. Is metabolic syndrome one of the risk factors for gallbladder polyps found by ultrasonography during health screening? Gut Liver 2007;1:138-44.

18. Chen CH, Lin CL, Kao CH. Association of gallbladder polyp and stroke: a nationwide, population-based study. Medicine 2015;94:e2192.

19. Cheng Y, Du CL, Hwang JJ, et al. Working hours, sleep duration and the risk of acute coronary heart disease: a case-control study of middle-aged men in Taiwan. Int J Cardiol 2014;171:419-22.

20. McClelland RL, Jorgensen NW, Budoff M, et al. 10year coronary heart disease risk prediction using coronary artery calcium and traditional risk factors: derivation in the MESA (Multi-Ethnic Study of Atherosclerosis) with validation in the HNR (Heinz Nixdorf Recall) study and the DHS (Dallas Heart Study). J Am Coll Cardiol 2015;66:1643-53.

21. Khera AV, Emdin CA, Drake I, et al. Genetic risk, adherence to a healthy lifestyle, and coronary disease. N Engl J Med 2016;375:2349-58.

22. Mozaffarian D, Benjamin EJ, Go AS, et al. Heart disease and stroke statistics-2016 update: a report from the American Heart Association. Circulation 2016;133:e38-360.

23. Kotseva K, Wood D, De Bacquer D, et al. EUROASPIRE IV: A European Society of Cardiology survey on the lifestyle, risk factor and therapeutic management of coronary patients from 24 European countries. Eur J Prev
Cardiol 2016;23:636-48.

24. McGovern PG, Jacobs DR Jr, Shahar E, et al. Trends in acute coronary heart disease mortality, morbidity, and medical care from 1985 through 1997: the Minnesota heart survey. Circulation 2001;104:19-24.

25. Knuth D. Seminumerical Algorithms. Boston: AddisonWesley, 1981.

26. Park SK, Miller KW. Random number generators: good ones are hard to find. Commun ACM 1988;31:1192-201.

27. Database NHIR. Taiwan. Available online: http://nhird. nhri.org.tw/en/Background.html

28. Méndez-Sánchez N, Tanimoto MA, Cobos E, et al. Cholesterolosis is not associated with high cholesterol levels in patients with and without gallstone disease. J Clin Gastroenterol 1997;25:518-21.

29. Kavanagh K, Davis MA, Zhang L, et al. Estrogen decreases atherosclerosis in part by reducing hepatic acylCo A: cholesterol acyltransferase 2 (ACAT2) in monkeys. Arterioscler Thromb Vasc Biol 2009;29:1471-7.

30. Juvonen T, Savolainen MJ, Kairaluoma MI, et al. Polymorphisms at apoB, apoA-I, and cholesteryl ester transfer protein gene loci in patients with gallbladder diseases. J Lipid Res 1995;36:804-12.

31. Li Q, Ge X, Xu X, et al. Comparison of the genes expression profiles between gallstones and gallbladder polyps. Int J Clin Exp Pathol 2014;7:8016-23.

32. Lin CJ, Lai CK, Kao MC, et al. Impact of cholesterol on disease progression. Biomedicine (Taipei) 2015;5:7.

33. Calle MC, Fernandez ML. Inflammation and type 2 diabetes. Diabetes Metab 2012;38:183-91.
Cite this article as: Chen $\mathrm{CH}$, Lin $\mathrm{CL}$, Kao $\mathrm{CH}$. The risk of coronary heart disease after diagnosis of gallbladder polyp: a retrospective nationwide population-based cohort study. Ann Transl Med 2019;7(23):753. doi: 10.21037/atm.2019.11.114 